\title{
EPIWORDS: A NEW ONLINE ENGLISH-RUSSIAN GLOSSARY OF MEDICAL, STATISTICAL, MICROBIOLOGICAL AND EPIDEMIOLOGICAL TERMS
}

Editorial team (eurosurveillance@ecdc.europa.eu) ${ }^{1}$

1. Eurosurveillance, European Centre for Disease Prevention and Control, Stockholm, Sweden

EpiNorth project had launched a new feature - EpiWords. EpiWords is a bilingual glossary (English - Russian) of medical, statistical, microbiological and epidemiological terms. It will help to diminish the terminology difficulties in professional communication among health care specialists using English and Russian language.

Currently, the EpiWords section contains more than 800 terms and word combinations in Russian and English and is freely available. The terms are arranged in alphabetical order. EpiWords is a complementary source and is not intended to replace medical information provided by other published or online dictionary/ glossary sources.

EpiNorth project staff plans to find and publish new terms in a timely manner and invites interested parties to send suggestions, comments or questions to EpiNorth project at epinorth@fhi.no

EpiWords can be accessed at the following link:

http://www.epinorth.org

This article was published on 11 September 2008.

Citation style for this article: Editorial team. EpiWords: a new online English-Russian glossary of medical, statistical, microbiological and epidemiological terms. Euro Surveill. 2008;13(37):pii=18980. Available online: http://www.eurosurveillance.org/ ViewArticle.aspx?ArticleId=18980 